\title{
Government Roadmap for IPv4 to IPv6 Network Migration: A Case of Nepal
}

\author{
Babu Ram Dawadi ${ }^{1}$, Subarna Shakya ${ }^{1}$, Ananda Raj Khanal ${ }^{2}$ \\ ${ }^{I}$ Department of Electronics and Computer Engineering, Pulchowk Campus, Tribhuvan University, Nepal \\ \{'baburd, $\left.{ }^{1} d r s s\right\} @ i o e . e d u . n p$ \\ ${ }^{2}$ Department of Engineering, Nepal Telecommunications Authority, Kathmandu, Nepal \\ corresponding author:arkhanal@nta.gov.np
}

\begin{abstract}
The recent breakthrough in Information and Communication Technologies(ICT) make the internet and e-mail as a basic service for the people of every country. The rapid growth in internet users and ICT market by the development of smart devices increase the challenges in hardware and networking management as well as efficiency on software and services. Hence migration to new and latest technologies like software defined networking, cloud computing and IPv6 addressing become inevitable for the service providers throughout the world. Asia is the first continent where shortage of IPv4 was faced. APNIC announced the depletion of IPv4 address on April 2011 [1]. Similarly the IPv4 pool on other Regional Internet Registries have already finished except AFRINIC. With the shortage of address, Asian countries like Japan, China, India, Malaysia etc... have developed the government policies and national roadmap. They are under monitoring and evaluation of the private and public networks. European union and the American government put forward the priority on IPv6 network migration. In this paper a migration roadmap for Nepal is being proposed after reviewing the world's IPv6 migration status, other countries policy and roadmap for migration planning as well as survey carried out with Nepalese telecom, internet market and government agencies .
\end{abstract}

Keywords: IPv4/IPv6, migration, roadmap, Stakeholder, Strategies

\section{Introduction}

Nepal is greatly influenced by the progress of the many countries in the region that had already welcomed the development of information technology (IT), particularly the two Asian countries, India and China, where Nepal is sandwiched in between. Hence Nepal is well aware of recognizing the importance of the production and use of information and communication technology (ICT) for competitiveness and growth and the need to incorporate national information infrastructures to sustain and expand its economies. we believe to make ICT accessible to everyone from urban to peri-urban to rural to far-rural places and for all disabled people, ethnic group, marginalized group, low income people and many more. This shall be achievable for the government, only if, infrastructures have sufficiently been deployed, proper technologies have been identified, suitable national policies, plan and roadmap have been formulated and the sufficient human resources have been developed.

It is the government responsibilities to make the national communication infrastructure well standard and competitive with global standards. While proper policy and regulatory framework is needed to cope with the private agencies towards the technology migration to make it synchronize 
with world's standard and fulfill the current demands of the market. Nepal gains significant momentum in country ICT development by encouraging involvement of private parties in the sector development through the liberalization in the telecommunication act. 2003 [2] with the announcement of new telecommunication policy in 2004 [3]. Similarly IT policy 2000 was aimed as a tool for development and growth. The IT policy was revised in 2010 to adapt with the new technological demands. Nepal broadband policy-2015 put higher priority in broadband infrastructure development and sharing as well as migration to latest networking infrastructure operable with IPv6. Broadband policy also focused on cloud based service provisioning [4]. The recently approved ICT policy-2015 focused with the vision for "Digital Nepal" to build knowledge based society by the involvement of private sectors as a public private partnership (PPP) for the development of national backbone networks as well as to uplift the ICT in all sectors of the Nepalese economy [5]. The Nepal government enterprise architecture [8] has proposed different networking infrastructure with hardware, software, applications and databases for the citizen centered services at all of its ministries and department. The security architecture has also been proposed with the enterprise architecture [9]. However operability of those resources with IPv6 addressing infrastructure is necessary to be ensured before the project completion. In this aspect, the latest tools and technologies to be required for the government system is required to research.

Due to shortage of 32 bits IPv4 address space and several issues exists with IPv4 operable network [10], several countries throughout the world have already planned for smooth migration to their country network into IPv6 operable network with the formation of migration roadmap, government task force, councils and national policies. Similarly network operators and service providers worldwide are in rush of migration to IPv6 network. Hence this paper recommend the national migration roadmap for the Government of Nepal to take necessary steps in IPv6 network migration.

\section{Literature Review}

\subsection{IPv6 Overview}

Within the last 20 years, the number of internet users reached more than 3 billion [11] and more than 7.2 billion mobile gadgets which is even bigger than world's population in 2015 [11]. The rapid growth of internet users and ICT business in the world led the exhaustion of IPv4 address space and forced us to move to new addressing scheme having 128 bits so called Internet Protocol version 6 (IPv6) [12]. In the next generation networking infrastructure, every connected device shall have more than one unique IPv6 addresses no matter how much the gadgets or internet users exist in the long future because 80 bits length is already astronomical where IPv6 is 128 bits long [12].

With the birth of new addressing scheme, the current issues of IPv4 addressing like limited address space, NAT proliferation, inefficient routing management (sometimes routing table overflows), inefficient and variable header length, lack of security and quality of service, IP header fragmentation leading to routing overhead and many more have been solved with IPv6. Hence IPv6 has the major features as classless addressing mechanism, standardized multicast addressing, address auto configuration (SLAAC) [13], new and optimized header format with fixed 40 bytes length, special header field like flow level for QoS, inbuilt security with IPSec framework, improvement on ICMPv6 [14] with the incorporation of ARP and IGMP like protocol, efficient and hierarchical routing infrastructure, oversize packet fragmentation by the IPv6 host itself instead of router to avoid unnecessary router processing and many more. 


\subsection{Government Initiative: International Scenarios}

In September 2000, Japan had put forward their National Strategy for the adoption of IPv6 called "u-Japan" (Ubiquitous Japan). It provided support for academic research through the Widely Integrated Distributed Environment (WIDE) project, development of new IPv6 based applications, and tax incentives for organizations that deploy IPv6. Then, in March 2001, Japan established the "e-Japan Priority Policy Program," which states that it would realize an Internet environment enabled with IPv6 by 2005 [15]. Japanese Telecommunications Company NTT became the world's first ISP to offer IPv6 services to the public. More than fifteen ISPs in Japan are providing commercial IPv6 services these days. Other Asian countries like Australia, China, India, South Korea, Singapore are those leading countries in Asia taking more concern on IPv6 network migration within the region. Similarly countries like Hong Kong, Thailand, Malaysia, Sri Lanka and Indonesia are at a nascent stage of IPv6 adoption and have got started on IPv6 initiatives with mandates for IPv6 transition setting target within the year 2016 [16].

India had already realized to the sensitivity of Network migration in 2004 and hence the Indian government put network migration into its main agenda. Department of Telecommunications (DoT) is taking the main role of this network migration in which India did policy decision on 2005 which includes workshop, training and awareness throughout the country, development of transition plan in consultation with stakeholders and creation of national IPv6 taskforce etc... as a continuity of this policy decision India published National IPv6 Deployment Roadmap Version I in 2010 [17] and second version of the roadmap in 2013 [18].

China, which is often held up as an example of a country that really needs IPv6 has protocol support of $49.5 \%$ [19] on IPv6. China Next Generation Internet project (CNGI), is a five-years plan with the objective of cornering a significant proportion of the internet space by implementing IPv6 early. China Telecom has started its IPv6 activities since 2003. The china IPv6 policy put the target to have mobile internet, cloud computing and other new businesses use IPv6. Similarly the new terminal supports IPv6 entirely and there will be 25 million IPv6 users beyond 2015 [20]. Similarly China Mobile is under the process to upgrade the entire network into IPv6 putting the targets of more than 3 million IPv6 users after 2016. It is promoting IPv6 in 3G and 4G mobile internet [20].

The Malaysian IPv6 council developed the IPv6 Roadmap as a strategic implementation plan for IPv6 in Malaysia and proposed target to make IPv6 enable at ISPs by 2006, at e-Government Network by 2008 and overall Malaysia in 2010. However it was revised in 2010 setting the target by 2015 to migrate completely to IPv6 enabled network in Malaysia. Malaysian Communications and Multimedia Commission (MCMC) is currently taking steps to make sure that all ISP's are ready to provide the IPv6 services [21]. The French IPv6 Task Force has put in strong participation for the deployment of IPv6. IPv6 has been deployed over internal IPv6 network between sites of France Telecom Research \& Development since 1998. France Telecom carrier has deployed an IPv6 native backbone to help customers to move forward [22]. The Austrian IPv6 Task Force was established in 2004. Austrian Academic Computer Network (ACONET), the Austrian NRN (National Research Network) first started experiments with IPv6 in the late 1990s. Since then ACONET has gone on to provide academic institutions within Austria with IPv6 services [23]. Later, Telecom Austria was involved in IPv6 related international projects namely Global Communication Architecture and protocols for new QoS services over IPv6 networks [24]. The National IPv6 working group (acted as Finish IPv6 Task Force) was established in 2002 and is supported by the Finnish Communications Regulatory Authority. The group promotes the adoption of the IPv6 protocol in the national communications network, and to monitor and examine the 
mechanisms necessary for the transition to IPv6 and the preparation of the related guidelines to ensure interoperability of communications networks, equipment and services [25]. In 2003, the German MoD (Ministry of Defense) had given guidelines and directives to all relevant organizations concerning the introduction of IPv6. The German IPv6 Task Force aims to provide leadership for the timely deployment and adoption of IPv6 in Germany [26]. UK IPv6 Council was established in April 2014 with the main objectives to create sharable platform for all stakeholders involved in IP related networks and business as well as collaborate with other organizations to make UK IPv6 activities visible to the world [27].

The U.S. Federal Government recognized the importance of transition to IPv6 in 2005 and generate the memorandum for transition planning to migrate the US federal backbone networks by 2008 . The Federal chief information officers (CIO) council published the second version of the roadmap for IPv6 adoption on July 2012 as a continuity and update on first version published on May 2009, including Federal IPv6 Taskforce with the purpose to help Federal Government agency leaders and industries leaders to successfully integrate IPv6 in the enterprises [28].

\section{IPv6 Readiness Status of Nepal}

A survey/questionnaire, interview were conducted with different stakeholders (eg: Major ISPs and Telcos, Government ministries and Departments) and various publications, reports were used to compile the qualitative and quantitative data and studied in this paper regarding the IPv6 migration status measurement of Nepal. The major information collected were core network devices/severs/ software operability status with IPv6, IPv4 address consumption status, reservation of IPv6 addresses, awareness/training activities, policy readiness status and human resource readiness for IPv6 operations.

ISPs and Telcos are the major stakeholders of Nepal. They provide ICT services to the government, industries, banks and other non-profit organizations. Nepal, after adopting the liberalization policy [3] to encourage involvement of private sectors in this field, there have been optimum competitions in telecom and ISP sectors which helped to reduce the digital divide and increase ICT accessibility to remote places. With the growing competition in the market constitutes the rigorous increase of ICT indicators. The graph in the fig. 1 below shows that the internet penetration has not that much of satisfactory that covers only $43.67 \%$ of the total population as compared with $98.35 \%$ voice penetration. Additionally internet accessibility is mostly confined to urban and peri-urban areas of Nepal, while it is facing lack of sufficient policy and lack of private enterprise interest towards IP network deployment in the rural zones.

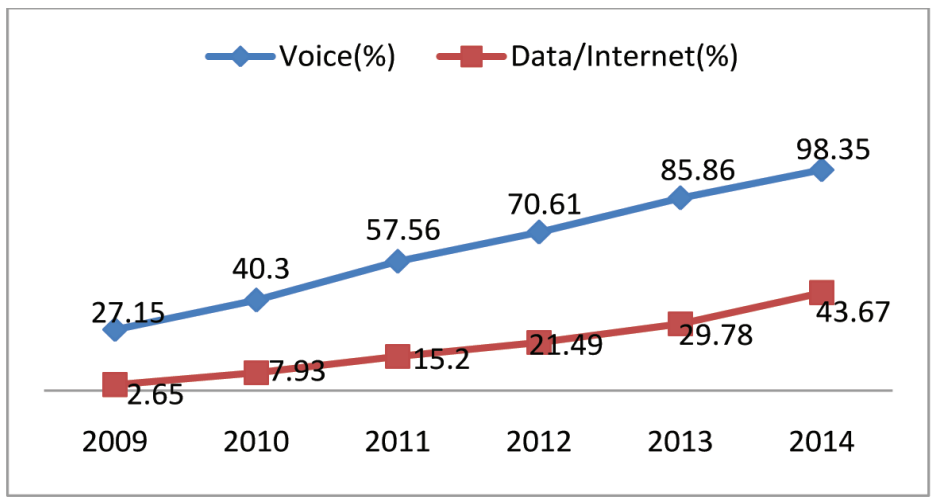

Fig. 1 Voice \& Internet Penetration of last six years[29] 
There are almost 46 ISPs in Nepal providing wired and wireless internet services with different schemes [29]. Almost 90\% ISPs are confined to city area especially to Kathmandu valley and the cities outside the capital. ISPs like World Link Communications Pvt. Ltd., Mercantile Communication Pvt. Ltd., Subishu Cablenet Pvt. Ltd., Vianet Pvt. Ltd., Websurfer Nepal Pvt. Ltd. are the major ISPs providing internet and email service at a greater scale. Nepal Telecom is the country's largest incumbent telecom operator covering the country by PSTN land lines as well as Mobile/CDMA services with broadband internet services like DSL/WiMAX/Fiber Lease Line and the 3G/GPRS service via mobile network. Similarly NCELL Pvt. Ltd. is the second largest mobile operator in the country, while there are in total six telecom operators providing mobile and basic telecom services under different licensing regimes [29].

\subsection{ISPs and Telcos Readiness Status}

From the survey (questionnaire, telephone conversations, interview) over the major ISPs, and Telcos of Nepal, we have highlighted some of the preliminary findings of the readiness status with respects to their network/infrastructure and its migration possibilities to IPv6.

Almost all ISPs are residing in the urban area provide internet service through Cable-TV, Fiber, Coax, and CAT6 as a wired mechanism. Wireless connection is also popular for home internet service. While some ISPs also offer MPLS data connectivity, Frame-relay and the VPN services. But Limited major ISPs (like Worldlink, Mercantile, WebSurfer, Vianet) have reserved IPv6 address block from the Regional Internet Registry. Major telecom service providers (Nepal Telecom, NCELL, UTL) have their core network almost ready to operate IPv6 and they put their priority to purchase IPv6 capable devices for their network during expansion. However they don't have solid plan of migration. Nepal Telecom is the only largest government incumbent operator has its core telecom network almost ready to operate IPv6 and is also in the process to purchase the AFT(NAT64) for IPv6 and IPv4 network inter communication. Almost all the ISPs of Nepal are well aware about the sensitivity of migration, however they don't have solid plan of migration. Most of the ISPs are marginally sustainable. The major issues for them are the cost of migration regarding the equipment costs, human resource \& capacity building. Major ISP's core network is almost dual stack capable and ready to enable into dual-stack mode within a year. However, due to the high cost of replacing CPEs at customer end is delaying the process of migration. A cost effective migration strategy is to be followed by the ISPs and Telcos of Nepal to upgrade the network in time. Government and stakeholder's coordination is important where government should proceed with best policy and roadmap. Government shall also provide special subsidy in the migration like tax exemption on IPv6 equipments like switches, servers and routers.

The internet users and organizations are not aware of the use of IPv6 in their services. ISPs are not in demand of IPv6 services to be offered by the customers. Uninterrupted service is the most for customer while some of the customers like banks, university network might have demand on IPv6 capable networks and applications. It is noticed that the knowledge and awareness about IPv6 and IPv6 ready equipments are minimal in the customer end, however the new equipments and software purchased are already IPv6 capable.

\subsection{Government Ministries and Department}

A mini survey had been carried out taking concerns of IPv6 migration in selected government ministries and departments. However the government technical officers (like computer officers/ engineers, IT directors) are aware of the technology, the non-technical higher level officers, those 
having decisive power at the government level are not aware of this technology and not convinced for the requirements of migration. ICT officers in the government ministries and departments are very limited in numbers that leads to compulsion of outsourcing ICT services to private agencies. There seems to be considerable skill gap in handling the ICT services and expanding the latest internet infrastructure in the government network due to the lack of sufficient training and awareness.

\section{Stakeholder Roles and Responsibilities}

Government is the main entity who can drives the network migration through policy directives. Various organizations directly and indirectly have significant roles and responsibilities to ensure smooth migration to IPv6 along with proper coordination with each others. The stakeholders apart from their general roles and functions might have a separate agenda towards the successful deployment of their own network into IPv6. The major stakeholders are depicted in the fig. 2.

The government ministries like Ministry of Information and Communication (MoIC) is the policy maker and has prominent role in deploying the IP network because all the infrastructures including private and public companies in telecom/ISP sector lies under MoIC with the significant role of Nepal Telecommunications Authority (NTA) as a regulator. NTA shall have major role in policy recommendation. National Information Technology Center (NITC) under Ministry of Science, Technology and Environment (MoSTE) \& Department of Information Technology (DoIT), is an ICT executing body has to have focus on government infrastructure deployment, operation and maintenance of different government owned servers and cloud system. It shall have leading role in deploying IPv6 in the government network. Ministry of Science and Technology, Ministry of Education and Universities have greater role in research and development as well as human resource development through the endorsement of relevant syllabus in the university course.

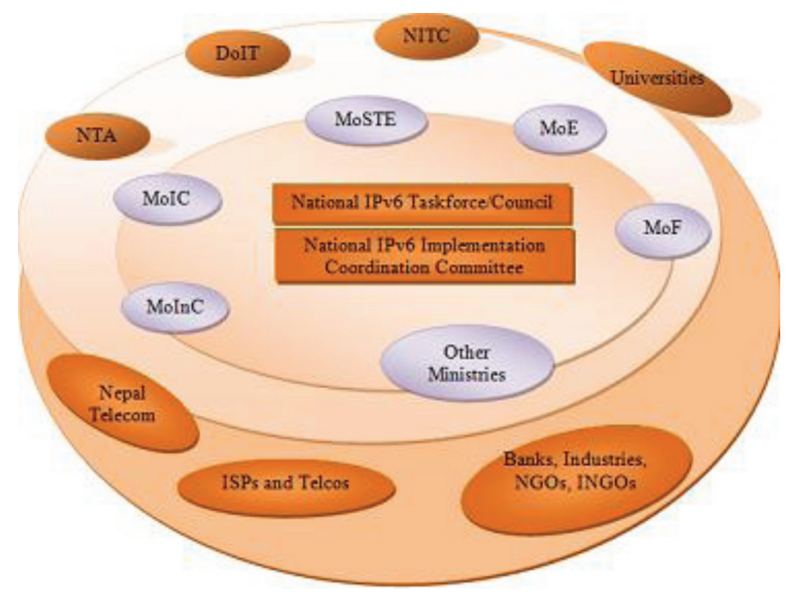

Fig.2 Major Stakeholders for Network Migration ${ }^{1}$

ISPs and Telcos are the main backbone of country network infrastructure. In this regards, special attention shall be required while enhancing IP network of those institutions in the country. Companies should be more responsible by themselves for their future sustainability to catch new technologies and be up to date keeping in line with the government, following necessary rules

1 MoIC: Ministry of Information and Communication, MoSTE: Ministry of Science \& Technology and Environment, MoE: Ministry of Education, MoF: Ministry of Finance, MoInC: Ministry of Industry and Commence, DoIT: Department of Information Technology, NTA: Nepal Telecommunications Authority, NITC: National Information Technology Center 
and regulations as well as they have prominent role to knock the government to facilitate for the change. Nepal Telecom (NT) is the government owned company provides Mobile, Landline and internet broadband services throughout the country. As being an incumbent operator, NT has biggest network in the country. Not only taking steps for its network migration, NT shall support other Telcos and ISPs for smooth migration and interconnection of the IPv6 network with its own test-bed network.

National IPv6 council (NIC) and National IPv6 implementation coordination committee (NIICC) are the two governing bodies proposed to carryout national network migration. NIC is a government steering body which shall form sub-steering committee and working groups for migration activities. Similarly NICC shall comprise of public and private stakeholders for all kinds of coordination among organizations in the country for successful migration to IPv6. The functionalities of NIC and NIICC are proposed in section 5 below on this paper.

Nepal Government has no any initiation taken from policy perspectives regarding the IPv6 network migration. Hence based on the survey, study and discussion, a migration roadmap has been proposed with this paper. However waiting for government policies and plan shall not be recommended for Telcos \& ISPs to move ahead with their migration because there are several transition mechanisms and strategies [12] already standardized. The proposed roles and responsibilities of government stakeholders in details are listed below:

\section{$\underline{\text { Responsibilities of MoIC }}$}

- Approve or proceed to cabinet approval of policies and strategies for migration of ISPs and Telcos network.

- Approve or proceed to approve policies and strategies for migration of broadcasting service network (Radio, TV)

- Ensure the proper rollout of IPv6 nationally through the Council.

- Lead for IPv6 pilot project implementation.

- Audit and monitor IPv6 implementation in nationwide government network.

\section{Responsibilities of MoSTE (DoIT)}

- Perform IPv6 research and development in coordination with Ministry of Education (MoE), Universities and other research networks.

- Coordinate for the nationwide IPv6 deployment.

- Organize conferences, research meetings and workshops for researchers, facilitating the sharing and dissemination of knowledge about IPv6.

- Organize awareness and training for the government resources regarding the technology migration in coordination with NITC.

\section{Responsibilities of NITC}

- Provides IPv6 training to government technical officers regarding IPv6 and its related technologies.

- Run the IPv6 pilot project for government network migration.

- Assess IPv6 readiness over government network.

- Migrate government e-portals, web-URLs, DNS/WEB and other government servers into IPv6 operable system. 
- Prepare guideline for .NP ccTLD server migration to IPv6 and its administration.

- Prepare migration plan to achieve the main target of migrating government network into IPv6 operable network.

\section{Responsibilities of NTA}

- Recommend policy framework/roadmap for network migration.

- Develop regulatory guideline for IPv6 deployment over the ISPs and Telcos networks.

- Conduct awareness, training and national workshop about IPv6 network migration.

- Implement migration plan for ISPs and Telcos.

- Assist government agencies and private organizations in migrating to IPv6.

- Recommend to council/taskforce if any the incentives that shall be provided to ISPs and Telcos to encourage for their network migration.

- Measure IPv6 readiness.

- Types approve the IPv6 enabled devices. Arrange for IPv6 certification.

- Regularly audit the ISPs and Telcos network to achieve the policy/roadmap implementation.

- Shall act as secretariat of NIICC and National IPv6 Task Force/Council.

- Prepare detailed plans, strategies and guidelines as a result of this Roadmap to enable implementation at all levels.

- Monitor the current migration process and recommend the new target in the roadmap.

\section{Responsibilities of MoE/Universities}

- Introduce courses on IPv6 (changing syllabuses; placing greater emphasis on IPv6) in the institutes of higher learning.

- Encourage more research work in IPv6.

- Collaborate with international research agencies and universities regarding IPv6 research and development.

- Introduce IPv6 into the appropriate courses in colleges and universities.

- Support certification courses in IPv6.

\section{Responsibilities of MoF}

- Ensure that all Government agencies adopt the 'Buy IPv6' policy.

- Shall take decision to provide tax incentives to companies that adopt IPv6.

- Shall take decision exempt IPv6 hardware and software from taxes.

- Allocate budget for government network migration.

\section{Migration Roadmap}

After the survey and study of major service providers of Nepal, we proposed the roadmap for IPv4 to IPv6 network migration. Roadmap mainly focused the steps with timeline required to be followed for smooth migration. The major targets are to be set at the beginning and based on this targets, all governmental and non-governmental stakeholders should follow the tasks in the roadmap defined below. 
- If $\mathrm{x}$ is the year of initiation for IPv6 migration,

Target 1: All ISPs and Telcos Network to be IPv6 enabled by the year $y(=x+2)$. (for example: year of initiation $\mathrm{x}=2016, \mathrm{y}=2016+2=2018$ is the target 1 ).

Target 2: All government and agencies networks to be IPv6 enabled by the year $z(=y+2)$.

Target 3: Full fledge IPv6 operation on any networks and commercial IPv6 services beyond the year $\mathrm{w}(=\mathrm{z}+2)$.

\section{The tasks in the roadmap are:}

1- Establish National IPv6 Implementation Coordination Committee (NIICC) comprising main stakeholders before the year of migration initiation. The committee shall have the following functionalities:

- Perform research on IPv6 network migration.

- Coordinates with ministries, departments and independent organizations to initiate for the migration.

- Assess the current IPv4 network infrastructure of the government, industries, banks, ISPs and Telcos.

- Organize stakeholder's workshop to get feedback on policy formulation/revision.

- Create multi-stakeholder working group including concerned departments and other stakeholders like ISPs, Telcos to track the migration during transition phase.

- Coordinate and initiate for working group formation to ISPs and Telcos with focal communication persons for migration to respond to the issues and update status.

- Organize bi-annual awareness generation program with concerned stakeholders.

- Initiate to create national IPv6 forum and coordinate with global IPv6 forum to get in touch with the migration and widespread the awareness generation through this forum.

- Identify the costs of migrating government networks and prepare long term migration budget.

- Assist the National IPv6 task force/council regarding all the concerned matters listed as well as policy formulation, plan review and other activities required for migration.

- Coordinate with international stakeholders like ITU, ICANN, IETF, APINIC, APT etc... regarding the migration.

- Proceed to approve national migration plan.

- Monitor and regulate the migration.

2- Establish National IPv6 Council (NIC) as an apex body under the chairmanship of concerned minister/state-minister including other stakeholder representatives for the main objectives of policy directions, leadership and overall implementation evaluation of network migration. The major tasks of NIC shall be as follows:

- Provide leadership and policy directions for nation-wide IPv6 deployment.

- Advice NIICC to carry out its functionalities.

- Assist National Planning Commission regarding budgetary allocation for government network migration.

- Approve or proceed to approve the necessary national policy, guideline, roadmap etc... regarding migration. 
- Create sub-committee and working group if required for smooth migration.

- Provide leadership to establish National Internet Registry (NIR) of Nepal.

- Regulate the government regulators to achieve the targets of migration in time as per the plan.

3- Plan for awareness and training/workshop of IPv6 and its migration for the requirement of appropriate human resources.

4- Reserve the IPv6 address block form RIR and establish small IPv6 test-bed network.

5- Assess the existing network for the dual stack operability measure.

6- Develop standards for IPv6 compliance device certification.

7- Interconnect the IPv6 test network among service providers and government/university research networks.

8- Migrate core network devices into dual stack mode.

9- Migrate servers and software/applications into dual stack mode.

10- Enable or replace service provider CPEs into dual stack mode.

11- Seek international transit for IPv6 services.

12- Turn off IPv4 stack in the network and choose appropriate appraoch (eg: dual stack lite, AFT, 464XLAT) for connection to IPv4 network/servers outside your network. Start IPv6 commercial services.

The tasks are mapped into the timeline so as to meet the targets specified on this roadmap.

(Assumed the year of inititation for IPv6 migration is 2016)

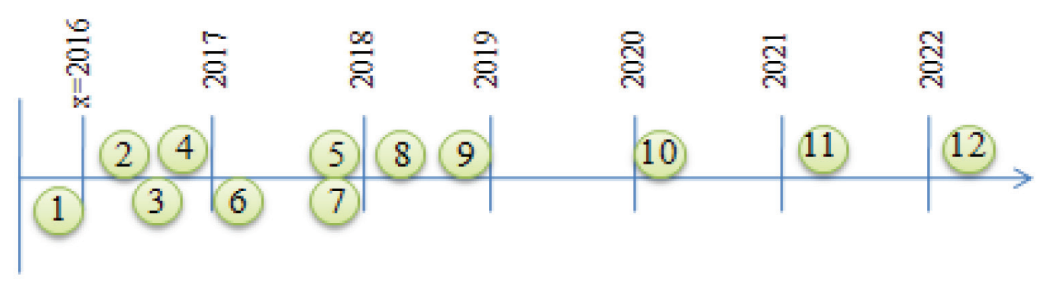

Fig. 3 Migration Roadmap Task Execution Targetted Timeline

Following table mentiones the responsible statkeholders to carryout the tasks defined in the roadmap.

TABLE 1: Tasks Matrix

\begin{tabular}{|l|l|}
\hline Tasks in the roadmap & Responsible Stakeholder(s) \\
\hline 1,2 & Government of Nepal, Initiation by NTA \\
\hline $3,4,5$ & All stakeholders for thier corresponding network. \\
\hline 6 & Nepal Telecommunications Authority \\
\hline $7,8,9,10,11,12$ & Service providers, Network Operators, NITC \\
\hline
\end{tabular}

\section{Conclusion}

Developing countries like Nepal are lacking behind the timely migration to IPv6 network. Government roadmap, policy framework are the major driving rules for proper migration to IPv6 in time. The survey of major ISPs and Telcos of Nepal shows that Nepalese stakeholders are not 
giving priority for network migration to IPv6. However the major service providers have reserved IPv6 from the APNIC and their core network is almost IPv6 ready but they have not delicately planned for the migration. Internationally the status of migration is in good condition, however the transition may prolong longer period of time. The internet users growth rate is increasing in Nepal. Hence considering the importance of government roles in network migration to IPv6, a research study of Nepalese internet and telecom market had been carried out and a migration roadmap with stakeholders roles and responsibilities have been proposed from this paper.

Acknowledgement: This research study was funded by Nepal Telecommunications Authority under the research grant "IPv6 Network Migration Roadmap of Nepal" carried out from February to August 2015.

\section{References}

[1] Apnic website, (2015). [online]. Available: http://labs. apnic.net/ipv4/report.html

[2] JPNIC(2006), Analysis and Recommendations on the Exhausting of IPv4 address space. [online]. Available: https://www.nic.ad.jp

[3] Government of Nepal (2004), Telecommunication Policy 2004. (M. o. Communication, Ed.). [online]. Available: from www.nta.gov.np/en/component/joomdoc/Policies/ TelecomPolicy_2004.pdf

[4] Mekonnen K and Abdulkadir T (2013), IPv6 Migration Framework - Case of Institutions in Ethiopia. HiLCoE Journal of Computer Science and Technology, 1(1), 565-572.

[5] Government of Nepal (2015), Information and Communication Technology (ICT) Policy-2015. [online] Available: http://www.nta.gov.np/en/component/joomdoc/ICT\%20Policy.pdf/download

[6] eGMP, e-Government Master Plan Consulting Report 2006.8 for Government of Nepal by Korean IT Industry Promotion Agency (KIPA). [online], Available: http://nitc.gov.np/download. php? $\mathrm{mod}=$ mydoc $\& \mathrm{f}=$ documents $\% 2 \mathrm{Fe}-\mathrm{GMP} . \mathrm{pdf}$

[7] KIPA website 2015, http://www.kipa.re.kr /language /main.jsp

[8] Government of Nepal (2006), Government Enterprise. [online], Available: http://nitc.gov.np / download.php?mod=mydoc \&f=documents2FNepal+GEA+Main+Report+v2.0.pdf

[9] Government of Nepal (2011), Government Enterprise Architecture Security Framework. [online], Available:http://nitc.gov.np/download. php?mod=mydoc $\& \mathrm{f}=$ documents\%2FNepal + GEA + Security + Architecture $+v 2.0 . p d f$

[10] Amer Nizar Abu Ali, (2012), Comparison study between IPV4 \& IPV6. International Journal of Computer Science Issues, 9(3), ISSN (Online): 1694-0814, www.IJCSI.org

[11] Gadgets usage statistics website, [online]. Available: http://www.independent.co.uk/lifestyle/gadgets-and-tech/news/there-are-officially-more-mobile-devices-than-people-in-theworld-9780518.html

[12] Dawadi BR, Joshi SR and Khanal AR (2015), Service Provider IPv6 Network Migration Strategies. Journal of Emerging Trends in Computing and Information Sciences, 6(10).

[13] Thomson S and Narten T (1998), IPv6 Stateless Address Auto-configuration, RFC2462

[14] Conta, A., \& Gupta, M. (2006). Internet control message protocol (icmpv6) for the internet protocol version 6 (ipv6) specification. RFC4443

[15] http://www.v6pc.jp/en/index.phtml 
[16] Sébastien Ziegler et all. (2013). IoT6 - Moving to an IPv6-Based Future IoT. FIA 2013, LNCS 7858, pp. 161-172, 2013

[17] Government of India, (2010). National IPv6 Deployment Roadmap Version I. [online], Available: http://www.dot. gov.in/sites/default/files /National-IPv6-Deployment-oadmap.pdf\%201.pdf

[18] Government of India, (2013). National IPv6 Deployment Roadmap Version II. [online], Available: http://www.dot. gov.in/sites/default/files /Roadmap\%20Version-II\%20 English\%20_1.pdf

[19] http://ipv6-test.com/stats/country/CN

[20] Zhiqiang, Li, (2013). IPv6 Development in China. [online]. Available: http://conference.apnic. net/ data/36/ipv6-in-china-lizhiqiang_1377575316.pdf

[21] MEWC, (2008). National Strategic IPv6 Roadmap. [online]. Available: http:/www.nav6.org/ Home /National\%20Strategic\%20IPv6\%20Roadmap\%20\%5BLast\%20Updated\%2010\%20 June $\% 202008 \% 5$ D.pdf

[22] European Commission IPv6 Portal website, [online], available: http://www.eu.ipv6tf.org / PublicDocuments/IPv6_Commercial_Deployment_in_Europe.pdf

[23] ACONET, [online]. Available: http://www.aco.net /ipv6.html?\&L=1

[24] IPv6 Cluster, IPv6 Research and Development in Europe, (2002). [online]. Available: http:// www.consulintel.es/pdf/ipv6_research_and_development_in_europe.pdf

[25] Finnish IPv6 Task Force, [online], available: http://www. fi.ipv6tf.org

[26] Danish IPv6 Taskforce, [online]. Available: http://www. ipv6tf.dk/home.

[27] UK IPv6 Council, [online]. Available : http://www.ipv6.org.uk

[28] U.S. IPv6 roadmap, (2012). Planning Guide/Roadmap Toward IPv6 Adoption within the U.S. Government. [online], Available: https://cio.gov/wp-content/uploads/downloads/2012/09 /2012_IPv6_Roadmap_FINAL_20120712.pdf

[29] NTA MIS Reports. [online]. Available: http://nta.gov.np/en/mis-reports-en 